\title{
A Notable Swarm Approach to Evolve Neural Network for Classification in Data Mining
}

\author{
Satchidananda Dehuri ${ }^{1}$, Bijan Bihari Mishra ${ }^{2}$ and Sung-Bae Cho ${ }^{1}$ \\ ${ }^{1}$ Soft Computing Laboratory, Department of Computer Science, \\ Yonsei University, 262 Seongsanno, Seodaemun-gu, Seoul 120-749, Korea. \\ Email:satchi.lapa@gmail.com, sbcho@cs.yonsei.ac.kr \\ ${ }^{2}$ Department of Computer Science and Engineering, College of Engineering Bhubaneswar, \\ Bhubaneswar-751024, INDIA. Email: misrabijan@gmail.com
}

\begin{abstract}
This paper presents a novel and notable swarm approach to evolve an optimal set of weights and architecture of a neural network for classification in data mining. In a distributed environment the proposed approach generates randomly multiple architectures competing with each other while fine-tuning their architectural loopholes to generate an optimum model with maximum classification accuracy. Aiming at better generalization ability, we analyze the use of particle swarm optimization (PSO) to evolve an optimal architecture with high classification accuracy. Experiments performed on benchmark datasets show that the performance of the proposed approach has good classification accuracy and generalization ability. Further, a comparative performance of the proposed model with other competing models is given to show its effectiveness in terms of classification accuracy.
\end{abstract}

Keywords: Particle Swarm Optimization, Evolutionary Computation, Genetic Algorithm, and Classification.

\section{Introduction}

Data mining [1] is an interdisciplinary field, whose core is at the intersection of machine learning, statistics, and databases. Data mining includes several tasks such as classification, association rule mining, clustering, regression, summarization etc. Each of these tasks can be regarded as a kind of problem to be solved by a data mining algorithm. Therefore, the first step in designing a data mining algorithm is to define which task the algorithm will address.

In this paper, we consider the problem of classification in data mining to validate our novel evolved neural network by particle swarm optimization. In general, a classifier partitions the feature space $\mathrm{X}$ into $C_{i}, i=1,2, \ldots, M$ classes such that: i) $C_{i} \neq \phi, i=1,2, . ., M$, ii) $\bigcup_{i=1}^{M} C_{i}=X$ and iii) $C_{j} \cap C_{k}=\phi, j \neq k, j=1(1) M, k=1(1) M$ (except for fuzzy classification domain) by constructing the hyper-planes or hyper-cubes. A hyper-plane can be written as: $d(x)=W \cdot X^{T}$, where $W=\left[w_{1}, w_{2}, \ldots, w_{N}\right]$ and 
$X=\left[x_{1}, x_{2}, \ldots, x_{N-1}, 1\right]$ are called the weight vector and augmented feature vector respectively. Now the problem is to classify an unknown sample based on the hyperplane.

Various-benchmark studies indicate that, the success of artificial neural networks (ANN's) for classification depends on the architecture and training of the network (i.e. searching an optimal sets of weights). A large number of techniques exist with which ANN's can be trained. Most applications use the back propagation (BP) algorithm or other training algorithms in the feed forward ANN's. But all these training algorithms assume a fixed ANN's architecture. There have been many attempts in designing ANN's architectures automatically, such as various constructive and pruning algorithms [2].

Design of a near optimal ANN's architecture can be formulated as a search problem in the network architecture and weight space where each point represents a type of architecture. Given some performance criteria, e.g., high classification accuracy, lowest architectural complexity, etc., the performance level of all architectures forms a surface in the space. Although genetic algorithm (GA) [3] is a better candidate for searching optimal neural network architecture but it can face the problem like permutation, noisy fitness evaluation, etc. [2].

This paper presents a novel method of evolution of artificial neural network using PSO [4] that not only evolves the set of weights but also evolves network architecture of low complexity. Compared with the GA, the PSO algorithm possesses some attractive properties, such as memory and constructive cooperation between individuals, which can avoid the permutation problem.

\section{Background and Related Work}

\subsection{Basics of PSO}

Particle swarm optimization (PSO), introduced by Kennedy and Eberhart [4] is a stochastic optimization technique mimicking the behavior of a flock of birds.

The algorithm presented below uses the global best and local best mechanism of PSO. Let $f: R^{n} \rightarrow R$ be the fitness function and there are $n$ particles, each with associated positions $x_{i} \in R^{n}$ and velocities $v_{i} \in R^{n}, i=1(1) n$. Let $\tilde{x}_{i}$ be the current best position of each particle and let $\tilde{g}$ be the global best.

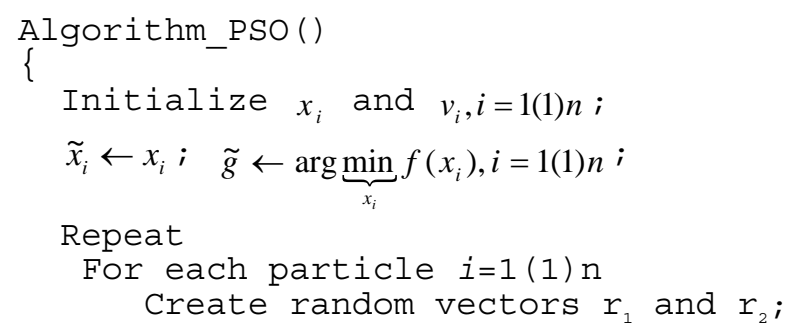




$$
\begin{aligned}
& \quad x_{i} \leftarrow x_{i}+v_{i} ; \\
& \quad v_{i} \leftarrow w v_{i}+c_{1}\left(r_{1} \otimes\left(\tilde{x}_{i}-x_{i}\right)\right)+c_{2}\left(r_{2} \otimes\left(\tilde{g}-x_{i}\right)\right) ; \\
& \quad \text { If } f\left(x_{i}\right)<f\left(\tilde{x}_{i}\right) ; \tilde{x}_{i} \leftarrow x_{i} ; \\
& \quad \text { If } f\left(x_{i}\right)<f(\tilde{g}) ; \tilde{g} \leftarrow x_{i} ; \\
& \text { End } \\
& \text { Until (stopping criterion met); }
\end{aligned}
$$

\subsection{Related Work}

$X$. Yao [2] seem to have been the first researcher for evolutionary neural network. He used a GA algorithm to evolve the architecture and weights of the network. Compared to GA, PSO algorithm possesses some attractive properties such as memory and constructive cooperation between individuals, so it has more chance to fly into the better solution areas more quickly and discover reasonable quality solution much faster. In PSO only few parameters are to be tuned. Unlike GA, the representations of the weights are easy and as there is no recombination and mutation operator, so there is a very negligible chance of facing the permutation problem. Further, as there is no selection operator in PSO, each individual in an original population has a corresponding partner in a new population. From the view of the diversity of population, this property is better than GA, so it can avoid the premature convergence and stagnation in GA to some extent.

Zhang et al. [5] has evolved PSO based ANN's and used in modeling product quality estimator for a fractionator of the hydrocracking unit in the oil refining industry. In their methodology, evolving ANN's architecture and weights are alternated and they used partial training for each individual architecture. The computation time of their proposed work is quite high due to the partial training.

Carvalho et al. [6] inspired by Zhang et al. works and then introduces the method PSO-PSO:WD based on the weight decay heuristic in the weight adjustment process in an attempt to obtain more generalization control.

Analyzing both algorithms we found that no one has given much emphasis on how many hidden layers required for the optimal architecture and nor even show about its optimality by experimental study. Further, neither given emphasis on how the particle is represented nor given much importance on numerical simulation to show the potentiality of their method. Further, as they are using PT algorithm for training so their method introduces overhead in computational time. Hence to cope with these problems we have motivated to develop a method, which can solve the said issues in addition to inheriting the basic characteristics.

\section{PSO for Evolving Swarm Network}

There are two major approaches to evolving the network architectures. One is the evolution of "pure" architecture with the randomly initialized connection weights, and 
then the connection weights will be trained after a near optimal architecture has been found. Another is the simultaneous evolution of both architectures and weights [2]. Unfortunately, the former brings out the noisy fitness evaluation that can mislead the evolution. Although the latter can effectively alleviate the noisy fitness evaluation problem, the nodes often become involved in a moving target problem because the solution space to be searched is too large. In general, it is the most significant reason why simultaneous optimization of the parameters of the entire networks can fail.

In order to solve preferably the above-mentioned problems, an approach is proposed where the number of hidden layers and number of neurons in the respective layer and set of weights are adaptively adjusted simultaneously.

In the proposed approach, both the architecture and the set of weights are encoded in particles and evolved simultaneously i.e. each particle represents a candidate solution of the architecture and weight space. In abstract view, Figure 1 shows the simultaneous evolution of architecture and weights.

1) Evaluate each particles based on the predefined criterion.

2) Find out the personal best $\left(p_{\text {best }}\right)$ of each particle and global best $\left(g_{\text {best }}\right)$ from the swarm.

3) Update particles velocity.

4) If the performance is satisfactory then stop, otherwise go to step 1.

Figure 1. Abstract View of Evolving Swarm Network

In its current implementations, swarm approach to evolving neural network is used to evolve feed forward neural networks with a characteristic function. We defined two characteristics functions-one is for 2-class problem and another is for 3-class problems. In the case of 2-class problem the characteristic functions is defined as: $f(o)=1$ if $o \leq \theta_{1}$, otherwise $f(o)=-1$, where $\theta_{1}$ is a threshold value and $o$ is the output produced by the output neuron. The positive value represent class 1 and the negative value represent class 2. Similarly, for three class problems the user has to choose two threshold values $\theta_{1}$ and $\theta_{2}$ based on that the characteristic function is defined as:

$$
f(0)=\left\{\begin{array}{ccc}
1 & \text { if } & o<\theta_{1} \\
0 & \text { if } & \theta_{1} \leq o<\theta_{2} \\
-1 & \text { if } & o \geq \theta_{2}
\end{array} .\right.
$$

In the proposed method we restrict ourselves to put one neuron in the output layer instead of putting neurons based on the number of classes. However, this is not an inherent constraint. In fact, the proposed approach has minimal constraint on the type of artificial neural networks, which may be evolved. The feed forward ANNs do not have to be strictly layered or fully connected between adjacent layers. Their may also contains hidden nodes with different transfer functions.

Let us see how the proposed approach is representing the particles as well as evaluating the fitness of each particle. 


\subsection{Particle Representation}

For representing the particles we have to set the protocols such as maximum number of hidden layers denoted as $\mathrm{L}_{\max }$, and maximum number of nodes for a particular hidden layer, denoted as $\mathrm{N}_{\max }$ a priori. Based on these values the particle can be represented as a vector notation, $P_{i}=\left\langle P_{i 1}, P_{i 2}, \ldots \ldots, P_{i b}\right\rangle$.

The first attribute $P_{\mathrm{i} 1}$ of the particle represent the number of hidden layers in the architecture. The value of $P_{\mathrm{i} 1}$ lies between 0 to $L_{\max }$, i.e. $0 \leq P_{i 1} \leq L_{\max }$. The feature from $P_{\mathrm{i} 2}$ to $P_{\mathrm{iLmax}+1}$ tells about the number of neurons in the respective hidden layer. The next features stores the weights between input layer and $1^{\text {st }}$ hidden layer and so on except the last feature of the particle $P_{\mathrm{ib}}$. The last feature i.e. $P_{\mathrm{ib}}$ stores the weight values of bias unit.

\subsection{Fitness evaluation}

The fitness of each particle of the proposed approach is solely determined by the classification accuracy based on the confusion matrix. Let the problem be a $C$ class problem. Then the confusion matrix for the $C$ class problem is defined as follows.

\begin{tabular}{|c|c|c|c|c|c|}
\hline & \multicolumn{5}{|c|}{ Predicted } \\
\hline \multirow{4}{*}{ Actual } & & $C_{1}$ & $C_{2}$ &. & $C_{\mathrm{C}}$ \\
\cline { 2 - 6 } & $C_{1}$ & $\mathrm{a}_{11}$ & $\mathrm{a}_{12}$ &. & $\mathrm{a}_{1 \mathrm{c}}$ \\
\cline { 2 - 7 } & $C_{2}$ & $\mathrm{a}_{21}$ & $\mathrm{a}_{22}$ &. & $\mathrm{a}_{2 \mathrm{c}}$ \\
\cline { 2 - 6 } &. &. &. &. &. \\
\cline { 2 - 6 } & $C_{\mathrm{C}}$ & $\mathrm{a}_{\mathrm{C} 1}$ & $\mathrm{a}_{\mathrm{c} 2}$ &. & $\mathrm{a}_{\mathrm{cc}}$ \\
\hline
\end{tabular}

The entries in the confusion matrix have the following meaning in the context of our study: $a_{11}$ is the number of correct predictions that an instance is $C_{1}, a_{21}$ is the number of incorrect predictions that an instance is $C_{2}$ and so on. The classification accuracy is measured by the following formula with a consideration of imbalance-ness of classes.

$$
\hat{f}_{C A}=\frac{1}{c}\left\langle\frac{a_{11}}{\sum_{j=1}^{c} a_{1 j}}+\frac{a_{22}}{\sum_{j=1}^{c} a_{2 j}}+\ldots \ldots . .+\frac{a_{c c}}{\sum_{j=1}^{c} a_{c j}}\right\rangle
$$

\section{Experimental Study}

The performance of the proposed model is evaluated using the four-benchmark databases taken from the UCI machine repository [8]. Table 1 presents a summary of the main features of each database that has been used in this study.

Table 1. Description of the features of the databases employed

Datasets Patterns Attributes Classes Patterns in Class1 Patterns in Class2 Patterns in Class3 


\begin{tabular}{ccccccc}
\hline IRIS & 150 & 4 & 3 & 50 & 50 & 50 \\
WINE & 178 & 13 & 3 & 71 & 59 & 48 \\
PIMA & 768 & 8 & 2 & 500 & 268 & - \\
BUPA & 345 & 6 & 2 & 145 & 200 & - \\
\hline
\end{tabular}

\subsection{Parameters Setup}

For simulation of the proposed models and to compare with other competing models like PSO-PSO:WD and PSO-PT, the different parameters considered for using the proposed method are presented in Table 2 . For all simulations we have used $g_{\text {best }}$ sociometry of PSO algorithm. Although this parameter area is quite restricted, and no systematic parameter optimization process has so far been attempted, however we set the parameters according to the suggested and widely used parameters in literatures [7] with a minor tuning such as $c_{1}=2.0476 \times w$ and $c_{C_{2}}=2.192 \times w$.

Table 2. Parameters required simulating the proposed method

\begin{tabular}{|c|c|c|c|}
\hline \multicolumn{4}{|c|}{ Parameters of the Proposed Method } \\
\hline \multicolumn{2}{|c|}{ PSO Parameters and Values } & \multicolumn{2}{|c|}{ Neural Network Parameter and Values } \\
\hline $\mathrm{W}$ & 0.729844 & $L_{\max }$ & 4 \\
\hline$c_{1}$ & 1.49445 & $N_{\max }$ & 10 \\
\hline$c_{2}$ & 1.6 & $\theta_{1}$ & 0.5 \\
\hline Population Size & 30 & $\theta_{2}$ & 0.833 \\
\hline \multicolumn{2}{|c|}{ Maximum Iterations } & & 5000 \\
\hline
\end{tabular}

\subsection{Cross Validation, Results and Discussion}

We have adopted the 2-fold cross-validation strategy with the intension of getting good classification accuracy. The dataset is randomly partitioned into two sets of equal size that are in turn used for building and testing the proposed model. While one part is used for building the proposed model the other part is used for testing the model, in a way that each one is used for opposite purpose. The percentage of correct classification for each dataset using the proposed model is presented in Table 3.

Table 3. Classification accuracy of proposed model

\begin{tabular}{ccccccc}
\hline Dataset & \multicolumn{2}{c}{ Hit Percentage in Training Sets } & \multicolumn{3}{c}{ Hit Percentage in Test Sets } \\
\cline { 2 - 7 } & Set1 & Set2 & Average & Set1 & Set2 & Average \\
\hline IRIS & 97.33 & 97.33 & 97.33 & 100.00 & 97.33 & 98.67 \\
WINE & 96.63 & 97.75 & 97.19 & 100.00 & 93.26 & 96.63 \\
PIMA & 73.44 & 75.26 & 74.35 & 85.42 & 82.81 & 84.16 \\
BUPA & 77.91 & 76.30 & 77.10 & 78.61 & 79.07 & 78.84 \\
\hline
\end{tabular}

With the same protocol the average comparative performance of the proposed 
method with its rival is presented in Table 4.

Table 4. Average comparative performance

\begin{tabular}{ccccccc}
\hline Dataset/ & \multicolumn{2}{c}{ Average Hit Percentage in Training Set } & \multicolumn{3}{c}{ Average Hit Percentage in Test Set } \\
\cline { 2 - 7 } Methods & $\begin{array}{c}\text { Proposed } \\
\text { Model }\end{array}$ & $\begin{array}{c}\text { PSO- } \\
\text { PSO:WD }\end{array}$ & PSO-PT & $\begin{array}{c}\text { Proposed } \\
\text { Model }\end{array}$ & $\begin{array}{c}\text { PSO- } \\
\text { PSO:WD }\end{array}$ & PSO-PT \\
\hline IRIS & $\mathbf{9 7 . 3 3}$ & 96.00 & 96.65 & $\mathbf{9 8 . 6 7}$ & 96.66 & 98.65 \\
WINE & $\mathbf{9 7 . 1 9}$ & 87.65 & 92.15 & $\mathbf{9 6 . 6 3}$ & 79.20 & 96.10 \\
PIMA & $\mathbf{7 7 . 1 0}$ & 60.25 & 72.75 & $\mathbf{7 8 . 8 4}$ & 58.55 & 76.25 \\
BUPA & $\mathbf{7 4 . 3 5}$ & 74.75 & 76.30 & $\mathbf{8 4 . 1 2}$ & 72.50 & 78.35 \\
\hline
\end{tabular}

From the results presented in Table 4 we can note that for all the datasets except BUPA the proposed method obtained better average classification accuracy in both training set and test set compared to the method PSO-PSO: WD and PSO-PT. In the case of BUPA even though the training performance of the proposed method is close or nearly better than other two methods but during testing the performance is quite better than other two methods.

The number of hidden layers and the number of neurons required in each hidden layer for the different dataset to generate the proposed architecture is presented in Figure 2.

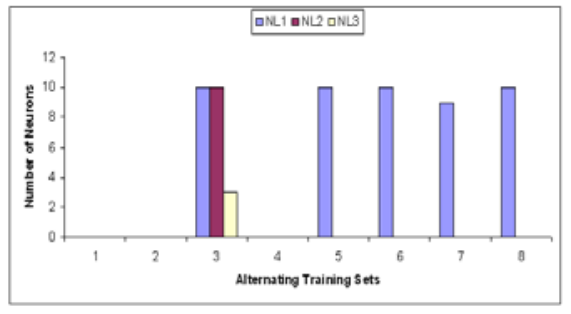

(a)

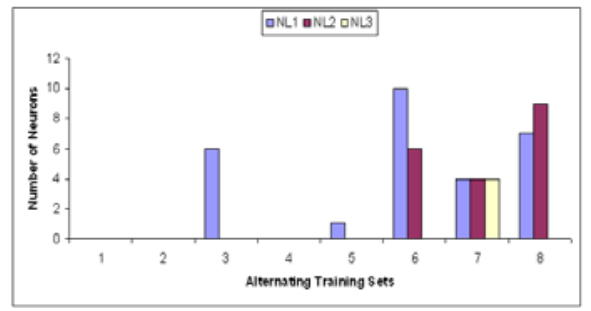

(b)

(c)

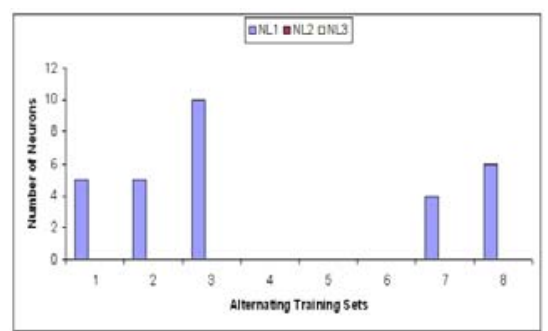

Figure 2. Architectural Complexity of (a) Proposed Method (b) PSO-PSO:WD and (c)PSO-PT

In view of the architectural complexity, on the whole the proposed approach is complex than other two models, whereas if we compare the classification accuracy the proposed approach is more promising than the other two approaches. Therefore, if one view from a multi-objective perspective no solution is better than others and it is 
also very difficult to optimize both these objectives simultaneously. These two objectives are very often conflicting to each other.

\section{Conclusions and Future Research}

In this paper, we have proposed a novel swarm approach to evolve neural network aiming to optimize simultaneously both architecture and set of weights. The proposed model is evaluated using the benchmark datasets considering the task of classification in data mining. Further, we compared our model with two other competing models such as PSO-PSO:WD and PSO-PT. Experimental studies demonstrated that the proposed model is quite superior to other two models in term of classification accuracy whereas in terms of architectural complexity our model introduces little overhead but can be tolerable by the data mining designer and ultimately decision maker. Future work should consist of experiments with other large datasets as well as the fine-tuning of the parameters used in the proposed method. It would be interesting to optimize the architectural complexity and classification accuracy considering as a multi-objective problem by PSO and generate the Pareto front.

Acknowledgments. The authors would like to thank the financial support of BK21 research program on Next Generation Mobile Software at Yonsei University, SOUTH KOREA.

\section{References}

1. Ghosh, A., Dehuri, S., Ghosh, S. (eds.): Multi-objective Evolutionary Algorithms for Knowledge Discovery from Databases. Springer-Verlag, Heidelberg, Germany (2008).

2. Yao, X., Liu, Y.: A New Evolutionary System for Evolving Artificial Neural Networks. IEEE Transactions on Neural Networks, vol. 8, no. 3, pp. 694-713 (1997).

3. Goldberg, D. E.: Genetic Algorithms in Search, Optimisation and Machine Learning. Addison-Wesley Pub. Co. (1989).

4. Kennedy, J., and Eberhart, R. C.: Particle Swarm Optimisation. In Proc. IEEE International Conference on Neural Networks, IEEE Service Center, Piscataway, NJ, pp. 39-43 (1995).

5. Zhang, C., Shao, H.: An ANN's Evolved by a New Evolutionary System and its Application. In Proc. of 39th IEEE Conference on Decision and Control, Sydney, pp. 3562-3563 (2000).

6. Carvalho, M., Ludermir, T. B.: Particle Swarm Optimisation of Neural Network Architectures and Weights. In Proc. of 7th International Conference on Hybrid Intelligent Systems, IEEE Computer Society Press, pp. 336-339 (2007).

7. Clerc, M., Kennedy, J.: The Particle Swarm-Explosion, Stability, and Convergence in a Multi-dimensional Complex Space. IEEE Transactions on Evolutionary Computation, vol. 6, no. 1, pp. 58-73 (2002).

8. Blake, C. L., Merz, C. J.: UCI Repository of Machine Learning Databases. http://www.ics.uci.edu/ mlearn/MLRepository.html. 